\title{
Blockade of miR-142-3p promotes anti- apoptotic and suppressive function by inducing KDM6A-mediated H3K27me3 demethylation in induced regulatory $\mathrm{T}$ cells
}

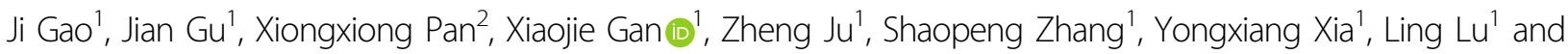 \\ Xuehao Wang
}

\begin{abstract}
In vitro induced human regulatory T cells (iTregs) have in vivo therapeutic utility. MicroRNAs (miRNAs) are a family of approximately 22-nucleotide non-coding RNAs that are processed from longer precursors by the RNases Drosha and Dicer. miRNAs regulate post-transcriptional protein expression through messenger RNA destabilization or translational silencing; miR-142-3p regulates natural Treg function through autophagy. We hypothesized that this miRNA may also have an iTreg regulation function. Antagomir-mediated knockdown of miR-142-3p improved Foxp3 (forkhead box P3) expression, regulatory function, cytokine expression, and apoptosis of iTregs in vitro, with or without inflammatory cytokine stimulation. miR-142-3p knockdown increased autophagy-related protein 16-1-mediated autophagy. Target prediction and luciferase assay results indicated that miR-142-3p binds directly to lysine demethylase 6A (KDM6A), which resulted in demethylation of $\mathrm{H} 3 \mathrm{~K} 27 \mathrm{me} 3$ and in turn upregulated expression of the anti-apoptotic protein $\mathrm{BCl}-2$. Based on these results, we propose a novel strategy that uses knockdown of miR-142-3p to enhance anti-apoptotic ability and function of iTregs by increasing KDM6A and $\mathrm{BCl}-2$ expression. This approach might be used as a treatment to control established chronic immune-mediated autoimmune and inflammatory diseases.
\end{abstract}

\section{Introduction}

Regulatory T cells (Tregs) are a subpopulation of T cells indispensable for maintenance of autoimmune tolerance ${ }^{1}$. They have critical roles in self-reactive lymphocyte suppression and mediation of immune homeostasis. The two major classes of Tregs are the thymic-derived Tregs (tTregs) and the in vitro induced Tregs (iTregs). Both classes have the same $\mathrm{CD} 4^{+} \mathrm{CD} 25^{+} \mathrm{CD} 127^{\text {low }}$ phenotype and express the transcription factor forkhead box P3

\footnotetext{
Correspondence: Ling Lu (Ivling@njmu.edu.cn) or

Xuehao Wang (wangxh@njmu.edu.cn)

${ }^{1}$ Hepatobiliary Center, First Affiliated Hospital, Nanjing Medical University, No.

300 Guangzhou Road, Nanjing, Jiangsu Province 210029, China

${ }^{2}$ Department of Anesthesiology, First Affiliated Hospital, Nanjing Medical

University, No. 300 Guangzhou Road, Nanjing, Jiangsu Province 210029, China

These authors contributed equally: Ji Gao, Jian Gu, Xiongxiong Pan

Edited by T. Kaufmann
}

(FOXP3). Studies using preclinical models and clinical trials found that Tregs prevent autoimmune disease and graft-versus-host disease (GVHD) ${ }^{2}$. The shortage of tTregs impedes the development of Treg therapy ${ }^{3}$. Use of adoptive transfer of iTregs has the potential because they have immune regulation functions similar to tTregs ${ }^{4}$. However, methods to enhance iTreg proliferation ability, survival, and function remain to be developed. In this study, we found for the first time that microRNA (miRNA) enhances the anti-apoptotic ability of iTregs through the mediation of histone modification.

Histone methylation is dynamically regulated by histone methyltransferase and demethylase to maintain gene activation and gene repression ${ }^{5}$. Trimethylation of H3K27 and $\mathrm{H} 4 \mathrm{~K} 20$ is associated with gene repression ${ }^{6}$. The antiapoptotic gene Bcl-2 is expressed in both effector T cells

\section{(c) The Author(s) 2019}

(c) (i) Open Access This article is licensed under a Creative Commons Attribution 4.0 International License, which permits use, sharing, adaptation, distribution and reproduction cc. in any medium or format, as long as you give appropriate credit to the original author(s) and the source, provide a link to the Creative Commons license, and indicate if changes were made. The images or other third party material in this article are included in the article's Creative Commons license, unless indicated otherwise in a credit line to the material. If material is not included in the article's Creative Commons license and your intended use is not permitted by statutory regulation or exceeds the permitted use, you will need to obtain permission directly from the copyright holder. To view a copy of this license, visit http://creativecommons.org/licenses/by/4.0/. 
and Tregs and is associated with anti-apoptotic ability and cell function ${ }^{7}$. Bcl-2 controls cell homeostasis of mouse iTregs ${ }^{8}$. Inhibition of histone demethylase decreases expression of $\mathrm{Bcl}-2$ by maintaining $\mathrm{H} 3 \mathrm{~K} 27 \mathrm{me} 3$ in the promoter region, which results in osteoblast apoptosis ${ }^{9}$. Lysine demethylase 6A (KDM6A) is also known as ubiquitously transcribed $\mathrm{X}$-chromosome tetratricopeptide repeat protein, which can specifically remove the methyl group from H3K27me3. KDM6A modulates $\mathrm{T}$ cell differentiation by modulating the methylation status of H3K27me ${ }^{10}$. Therefore, we hypothesize that KDM6A can improve the biological activity of iTregs by targeting histone demethylase to regulate histone methylation.

miRNAs are a family of small non-coding RNAs that can target messenger RNA (mRNA) transcription or mediate post-transcriptional gene repression with a short seed region complementary to mRNA sequences. miRNAs positively or negatively instruct the differentiation and suppression function of iTregs ${ }^{11}$. miR-142-3p can negatively regulate $\mathrm{T}$ cell activation in patients with systemic lupus erythematosus (SLE) ${ }^{12}$. Knockdown of miR$142-3 p$ results in better proliferation and immunosuppressive ability by targeting autophagy through upregulation of autophagy-related protein 16-1 (ATG16L1) in tTreg $^{13}$. Thus, our objective was to determine whether miR-142-3p can also regulate iTreg proliferation, survival, and immunosuppression. We were the first to find that knockdown of miR-142-3p enhanced iTreg anti-apoptotic ability and suppressive function by increasing $\mathrm{Bcl}-2$ expression through promoting H3K27me3 demethylation by targeting KDM6A, both in vitro and in vivo.

\section{Materials and methods Mice}

NOD CRISPR Prkdc Il2r gamma (NCG) mice, highly immunodeficient mouse, were purchased from Model Animal Research Center of Nanjing University, and housed in a specific pathogen-free facility with up to 5 mice per micro-isolator cages. All the mice were female and used at 6-8 weeks. Animal protocols were approved by Nanjing Medical University.

\section{Cell purification and culture}

Human peripheral blood (PB) leukapheresis products of volunteers were obtained from the Department of Hematology in the Affiliated Jiangning Hospital of Nanjing Medical University. Naive human PB T cells $\left(C D 4^{+} \mathrm{CD} 45 \mathrm{RA}^{+}\right)$ were sort purified from $\mathrm{PB}$ mononuclear cells (PBMCs) (Ficoll-Hypaque, Amersham Biosciences) by magneticactivated cell sorting (MACS pro) (Miltenyi Biotec, Germany) in a two-step procedure of magnetic beads sorting.

Naive T cells were induced to iTregs with anti-CD3/ CD28 mAb-coated Dynabeads (Life Technologies,
Carlsbad, CA, USA) at 1:1 (cell-to-bead) ratios in the presence of tumor growth factor- $\beta$ (TGF- $\beta)(1 \mathrm{ng} / \mathrm{ml})$ (Bio-Techne, Abingdon, OX, USA) and recombinant interleukin-2 (IL-2) $(100 \mathrm{U} / \mathrm{ml})$ (Chiron, Emeryville, CA, USA) in X-Vivo-15 (BioWhittaker, Walkersville, MD, USA) media supplemented with $10 \%$ fetal bovine serum (Valley Biomedical) for $72 \mathrm{~h}$.

The iTregs were cultured in the same media with the addition of recombinant IL-2 $(300 \mathrm{U} / \mathrm{ml})$ at the concentration of $0.5 \times 10^{6}$ cells $/ \mathrm{ml}$. IL-2 $(300 \mathrm{U} / \mathrm{ml})$ was added every 2 or 3 days. iTregs were treated with $\mathrm{miR}$ inhibitor or miR mimic (Ribobio Corporation, Guangzhou, China) and renewed together with IL-2 on point days. The inhibitor group was treated with miR-142-3p inhibitor $(100 \mathrm{nM})$, while the mimic group was treated with miR-142-3p mimic ( $50 \mathrm{nM})$. Cells were collected and tested as described.

\section{Flow cytometry, ImageStream, and antibodies}

Human-specific monoclonal antibodies used for flow cytometry included CD4 (PE-CY7), CD8 (APC), CD25 (APC-CY7), CD45RA (BV421), HLA-A2 (PE), Annexin V (Pacific blue), propidium iodide (PI), Ki67 (PE), and Bcl-2 (APC) purchased from BioLegend, and Foxp3 (APC) and CD127 (FITC) purchased from BD Pharmingen. Among them, Ki67 (PE), Bcl-2 (APC), and Foxp3 (APC) were used for intracellular flow cytometer assay, while the others needed surface staining. Tumor necrosis factor- $\alpha$ (TNF- $\alpha)$ (FITC), interferon- $\gamma$ (IFN- $\gamma$ ) (APC), TGF- $\beta$ (FITC), and IL-10 (PE), human-specific antibodies for ICFC purchased from BioLegend, were used to detect cytokine secretion in iTregs. Annexin V (PE)/PI were applied to detect the cell apoptosis of iTreg. Sample acquisition was performed by a CATON II (BD Bioscience) and data were analyzed with FlowJo VII software (TreeStar).

\section{Anti- and pro-apoptotic gene expression analysis}

RNA was collected from cell samples using EasyPure RNA Kit Mini (TransGen Biotech; Beijing, China). Complementary DNA (cDNA) synthesis was completed as described in the TransScrip First-Strand cDNA Synthesis SuperMix (TransGen Biotech; Beijing, China). Control gene used glyceraldehyde 3-phosphate dehydrogenase, and related gene expression (Bcl-2, Mcl-1, BID, BAD, and BAX) (all from IDT, Coralville, IA, USA) were tested on an Applied Biosystems 7500 Real-Time PCR System using Fast SYBR Green Master Mix (\#4385612) and Assay on Demand primer/probe Kits (Applied Biosystems, Waltham, MA, USA). The results were further analyzed to acquire the average delta CT. PCR cycling conditions were performed as follows: $94{ }^{\circ} \mathrm{C}$ for $5 \mathrm{~s}$ and $60^{\circ} \mathrm{C}$ for $30 \mathrm{~s}, 40$ cycles and then $95^{\circ} \mathrm{C}$ for $10 \mathrm{~min}$. 


\section{miRNA target prediction and luciferase assay}

Potential consequential pairing of target region and miRNA was predicted by miRNA prediction software microRNA (http://www.microrna.org/), TargetScan (targetscan.org), and MIRDB (http://www.mirdb.org/). Plasmid transfections for luciferase assays in 293T cells were performed with $0.1 \mu \mathrm{g}$ of firefly luciferase plasmid with the wild-type or mutated (mut) 3'-UTR sequences of KDM6A and $0.4 \mu \mathrm{g}$ miR-142-3p precursor or negative control precursor along with Renilla luciferase in a 24-well plate using Lipofectamine 2000 (LP2000) as described by the manufacturer. Luciferase activity was measured $48 \mathrm{~h}$ post transfection using the Dual Luciferase Reporter Assay System as described by the manufacturer (Promega). The relative expression of luciferase in the miRNA-NC group was homogenized to 1 in the two groups transfected with the same luciferase plasmid. The ratio of the target miRNA group to the miRNA-NC group indicates the relative expression of luciferase. Data are mean and standard deviation (SD) of separate transfections.

\section{Suppression assays}

The suppressive function of iTreg cultured in vitro was examined with a carboxyfluorescein succinimidyl ester (CFSE) inhibition assay as previously published ${ }^{14}$. Briefly, purified PBMCs were labeled with CFSE (Invitrogen) and stimulated with anti-CD3 mAb-coated beads (Dynal) \pm cultured iTreg (1:2 to 1:16 iTregs/PBMCs). Four days later, cells were harvested and stained with CD4 and CD8 antibodies. Suppression was accounted from the Division Index (FlowJo, TreeStar). iTregs suppressed CD4 and CD8 $\mathrm{T}$ cell responses equivalently and only CD8 data are presented.

\section{Xenogeneic GVHD model}

NCG mice between 6 and 8 weeks old were housed up to 5 per micro-isolator cage in a specific pathogen-free facility. On day 0 , mice were irradiated with 150 cGys. Human PBMCs $\left(10 \times 10^{6}\right)$ were transferred with or without iTregs $\left(10 \times 10^{6}\right)$ treated or untreated. Clinical scores were accounted based on GVHD symptoms of mice recorded daily. Each mouse was weighed thrice weekly. Human PBMC and iTreg in PB were detected by flow cytometry on the specified dates.

\section{Pathological examination}

Independent xenogeneic graft-versus-host disease (xGVHD) model experiments were performed as described above. Mice were sacrificed to obtain and make organ paraffin specimens on day 21. Hematoxylin-eosin (HE) staining on paraffin specimens was used to assess tissue damage and inflammatory changes. Immunofluorescence was performed with primary human monoclonal antibodies HLA-A2 and Foxp3, and staining with secondary antibodies Donkey anti-Mouse IgG $(\mathrm{H}+\mathrm{L})$ Highly CrossAdsorbed Secondary Antibody Alexa Fluor 488 and Donkey anti-Goat IgG $(\mathrm{H}+\mathrm{L})$ Cross-Adsorbed Secondary Antibody Alexa Fluor 647 (all antibodies from Invitrogen), corresponding to HLA-A2 and Foxp3, respectively. The ImageJ software was used to analyze all quantitative images. To quantify the positive cells, images in the same series were changed to type of RGB stack and adjusted to the same threshold. Then, the "analyze particle" option of ImageJ was used to detect the positive fluorescence intensity, indicating the HLA-A2+ or Foxp3 + cell proportion.

\section{Statistical analysis}

Reverse transcription-polymerase chain reaction (RTPCR) data were analyzed using the SDS v2.3 software. Survival data were analyzed using Prism 5 (Mantel-Cox). Other data were analyzed by analysis of variance or Student's $t$ test. Probability $(P)$ values $\leq 0.05$ were considered statistically significant.

\section{Results}

miR-142-3p regulates the proliferation, Foxp3 expression, and function of iTregs in vitro

Initially, we collected CD4+CD45RA+CD25- naive $\mathrm{T}$ cells (purity $295 \%$ ) from healthy donor PB samples. Naive T cells were then induced into iTregs. We observed in vitro changes in iTreg numbers and expansion for 16 days after the induction. The fold expansion reached a peak after 6-8 days of culture and then gradually decreased (Fig. 1a, b).

miR-142-3p genetic variations are associated with inflammatory bowel diseases ${ }^{15}$. We also previously found that miR-142-3p can negatively regulate tTreg proliferation, Foxp3 expression, and function in vitro ${ }^{13}$. Therefore, we also examined whether miR-142-3p controls iTreg biological properties. iTreg expansion decreased after day 7, so the inhibitor or mimic was added to the iTregs on day 7 and cell numbers and expansion were monitored. We found that knockdown of miR-142-3p promoted expansion of iTreg in vitro (Fig. 1c, d).

Next, we quantified the protein expression of Foxp3 and the suppressive function of iTregs after treatment. Knockdown of miR-142-3p using an inhibitor increased Foxp3 expression in the iTregs; treatment with a miR142-3p mimic had no effect (Fig. 1e, f). Subsequently, we performed the suppression assay using CFSE staining. The inhibitor group suppressed CD8 $+\mathrm{T}$ cell proliferation via enhanced suppressive function (Fig. 1i). The mimic group did not affect Foxp3 expression. The suppressive function was downregulated, which indicated that the overexpression of miR-142-3p negatively controlled iTreg function via other mechanisms. 

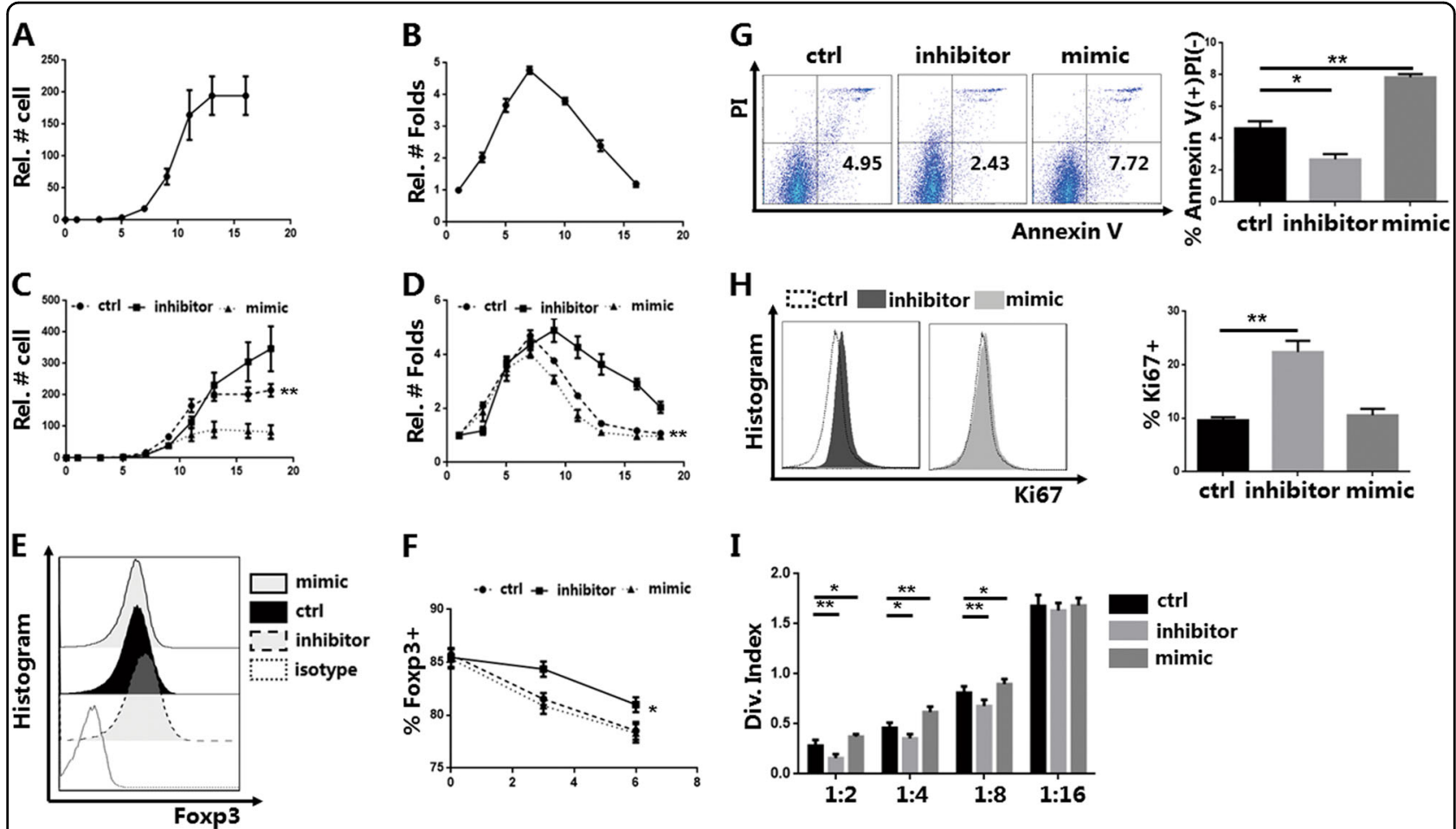

$\mathbf{F}$

I
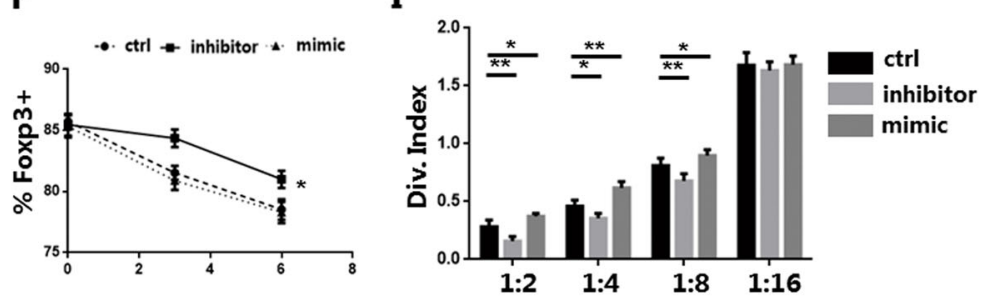

Fig. 1 Knockdown of miR-142-3p improves the proliferation, apoptosis, and function of in vitro induced Tregs (iTregs) in vitro ( $n=3)$. CD4 +CD45RA+-naive T cells were sort purified using magnetic-activated cell sorting and induced as CD4+CD25+CD127-FOXP3+iTregs, which were stimulated with anti-CD3/28 beads in the presence of fetal bovine serum (FBS) and interleukin-2 (IL-2). The iTregs were then harvested after treatment with a miR-142-3p inhibitor or mimic for 3 days on day 7. a Cell counts and $\mathbf{b}$ relative folds of expansion were recorded every 2 to 3 days. $\mathbf{c}$, $\mathbf{d}$ Cell counts and relative folds of expansion of iTregs treated with miR-142-3p inhibitor or mimic were recorded every 2 to 3 days and compared to the control cells. e Flow detection and expression of forkhead box P3 (Foxp3) in iTregs treated with an inhibitor or mimic on day 10 (gated on CD4 +CD127- cells). Representative result. f Foxp3 expression in iTregs of each group was recorded at different time points (days 7, 10, and 13). g Cell apoptosis assay using Annexin V/PI (propidium iodide) staining and \% Annexin V+ PI- of iTregs from each group (gated on CD4+CD127- cells). Representative result. $\mathbf{h}$ Flow detection and positive proportion of Ki67 staining of iTregs from each group (gated on CD4+CD127- cells).

Representative result. i Division index for CD8+ T cell mediated with anti-CD3 proliferation in vitro, at ratios from 1:2 to 1:16 (iTreg: peripheral blood mononuclear cells) as detected using carboxyfluorescein succinimidyl ester dye dilution. Mean \pm standard error of the mean values are presented. ${ }^{*} P$ $<0.05$ and ${ }^{*} P<0.01$

We also examined the expression of Ki67 to determine whether miR-142-3p controlled the proliferation of iTregs and tested cell apoptosis using Annexin-V staining with PI. After the inhibitor treatment, Ki67 expression was increased and the early apoptosis was decreased. In contrast, treatment of the mimic increased the early apoptosis of iTregs. Similar to the results for Foxp3, overexpression of miR-142-3p had no effect on Ki67 expression (Fig. 1g, h).

\section{Knockdown of miR-142-3p upregulates the proliferation, Foxp3 expression, and function of iTregs under inflammatory conditions}

During complex disease conditions the iTreg is required to play an immunomodulatory role in the development of inflammation ${ }^{16}$. Therefore, we simulated the inflammatory environment in vitro by adding IL- $1 \beta$ and IL- 6 . Thus, we detected whether the iTregs were still affected by miR- 142-3p under inflammatory conditions. Both IL-1 $\beta$ and IL-6 were added to each group for $24 \mathrm{~h}$ to simulate an inflammatory environment.

We then collected the iTregs and examined Foxp3 expression using flow cytometry. Knockdown of miR-1423p still upregulated the expression of Foxp3; there were no changes in the mimic group (Fig. 2a, b). Concurrently, based on the results of early apoptosis measured using Annexin V/PI staining, we found that each group affected by inflammation had increased cell apoptosis (Fig. 2c, d). However, when treated using a miR-142-3p inhibitor, the increased early inflammation-induced apoptosis was attenuated; overexpression of miR-142-3p worsened the inflammation. Expression of Ki67 was promoted with a miR-142-3p inhibitor (Fig. 2e, f).

Consistent with the Foxp3 expression result, knockdown of miR-142-3p enhanced the suppressive ability of the iTregs. Similar to normal culture conditions, 


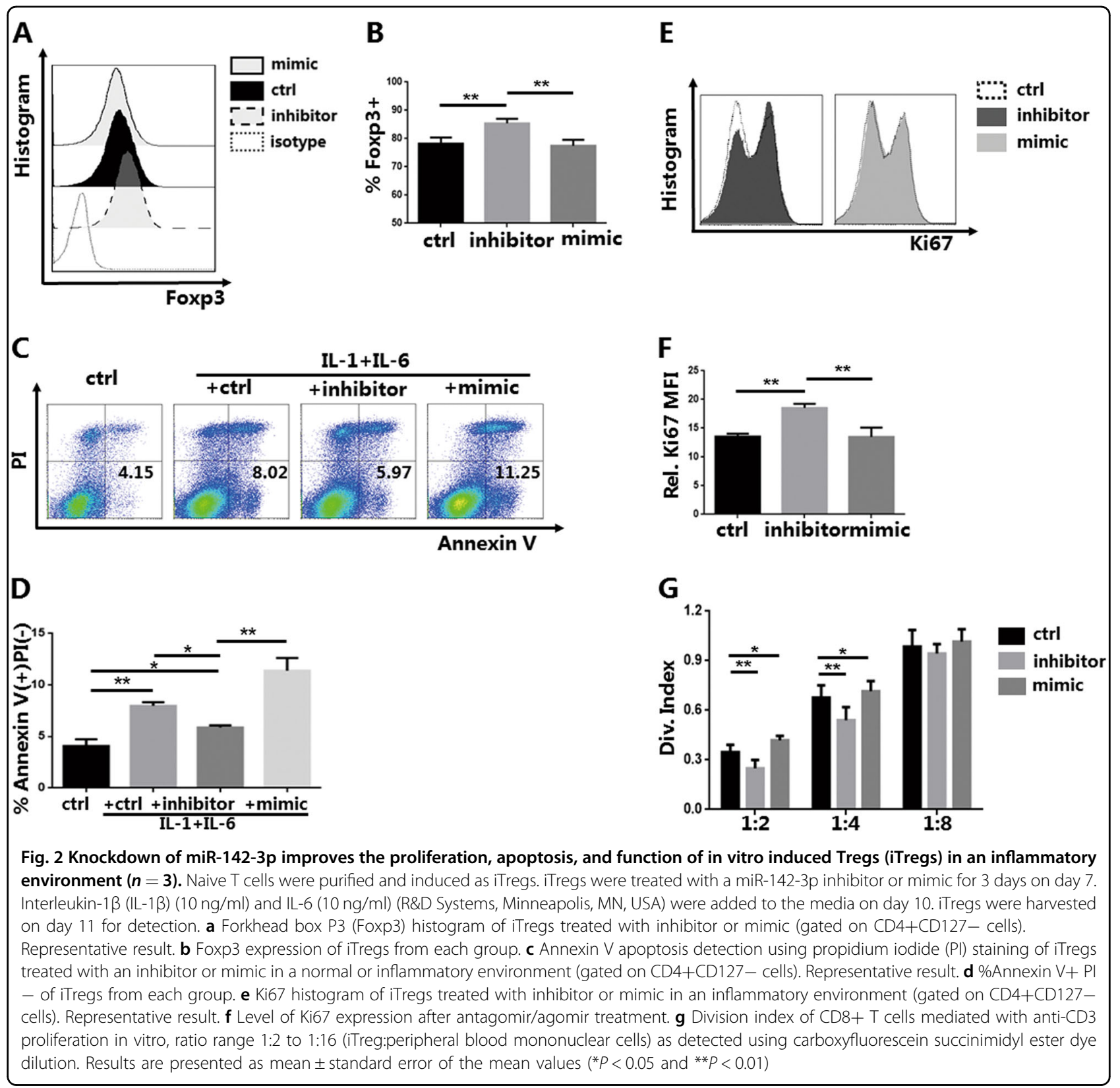

overexpression of miR-142-3p did not affect Foxp3 expression, but it weakened iTreg-suppressive function (Fig. 2f).

miR-142-3p affects the secretion of anti-inflammatory and pro-inflammatory cytokines in iTregs

Unlike tTregs, the mechanism by which iTregs have an immunoregulatory role is mainly through the secretion of various cytokines, in addition to the core protein Foxp $3^{17,18}$. A series of cytokine experiments found that cytokines are associated with iTreg function and differentiation $^{19,20}$. Therefore, we examined whether downregulation or over-expression of $\mathrm{miR}-142-3 \mathrm{p}$ affected
iTreg function through regulation of cytokine secretion. TGF- $\beta$ and IL-10 can exert immunosuppressive effects ${ }^{21}$. Neither TGF- $\beta$ nor IL-10 secretion changed with the miR-142-3p inhibitor treatment, compared with the control group. But overexpression of miR-142-3p resulted in significant decreases in TGF- $\beta$ and IL-10 secretion (Fig. 3a, d, e).

TNF- $\alpha$ enhances CD8 $+\mathrm{T}$ cell function via activation and proliferation pathways; apoptosis and destruction of GVHD target tissue results ${ }^{22-24}$. IFN- $\gamma$ can enhance the role of TNF- $\alpha$ in pro-inflammatory factors and increase TNF secretion in cultured macrophages ${ }^{25,26}$. TNF- $\alpha$ and IFN- $\gamma$ did not change in the mimic group. However, 


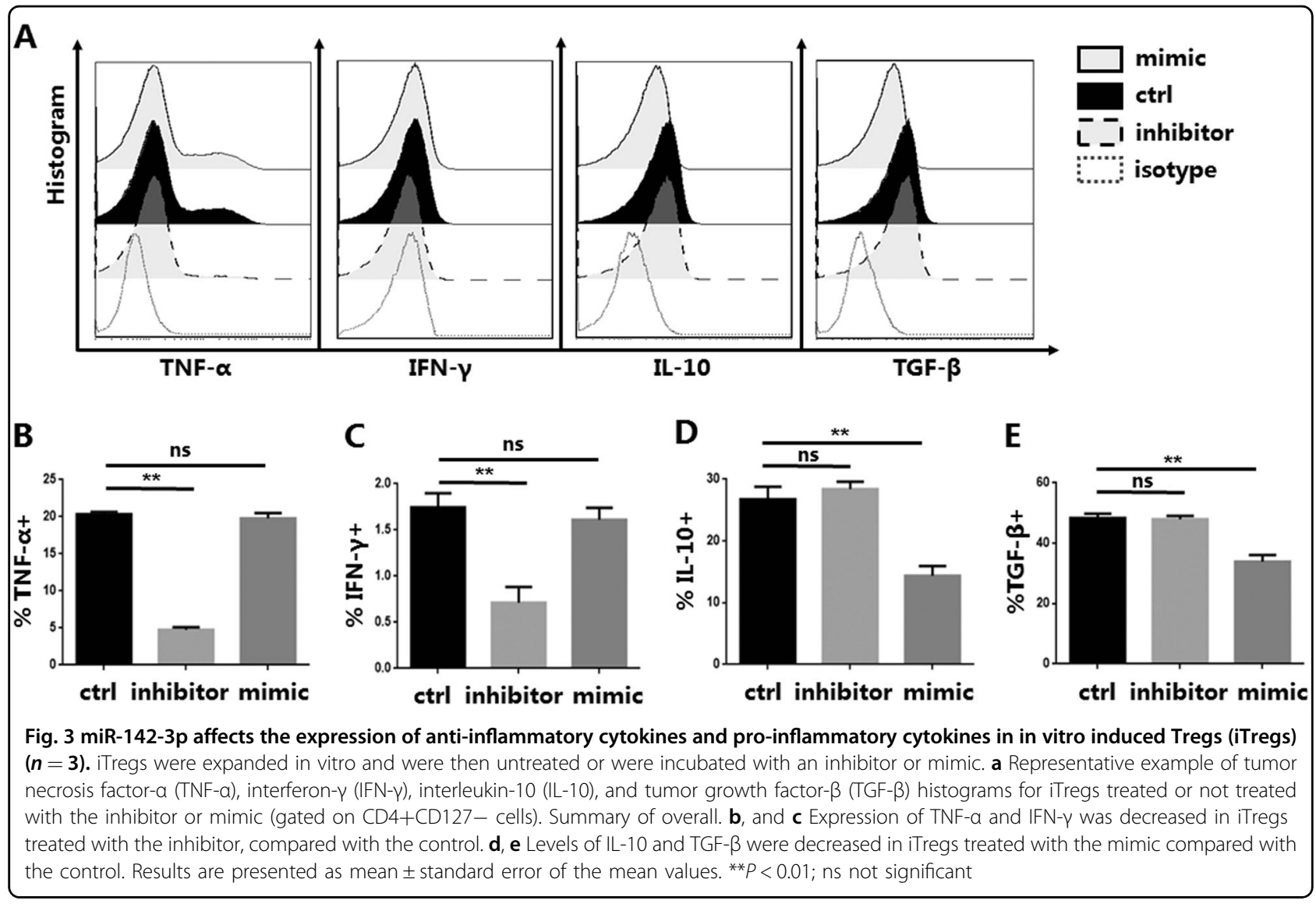

knockdown of miR-142-3p significantly reduced TNF- $\alpha$ and IFN- $\gamma$ secretion (Fig. 3b, c). Other cytokines, IL-2, IL4 , and IL-17, did not differ between the three groups (data not shown). Taken together, these results indicated that overexpression of miR-142-3p reduced the inhibitory function of iTregs by affecting cell survival and decreasing anti-inflammatory cytokine production.

\section{Knockdown of miR-142-3p increases ATG16L1 mRNA and ATG16L1 protein expression in iTregs}

Knockdown of miR-142-3p did attenuate iTreg apoptosis. However, cell survival is associated with a variety of factors, including autophagy. Autophagy is associated with the survival and differentiation of $\mathrm{T}$ cells ${ }^{27,28}$. Our previous study found that tTreg autophagy status and ATG expression changes during the process of expansion in vitro. Therefore, we examined autophagy-related proteins associated with the survival of lymphocytes (i.e., ATG3, ATG5, ATG7, and ATG16L1) ${ }^{29-32}$, and only ATG16L1 decreased during iTreg expansion (Fig. 4a, b). Therefore, on day 7 we treated iTregs with an inhibitor or mimic of miR-142-3p for 3 days. Using western blot and quantitative RT-PCR (qRT-PCR), we then detected ATG16L1 protein and mRNA expression, respectively. Knockdown of miR-142-3p increased both ATG16L1 protein and mRNA expression; overexpression of miR142-3p had an inverse effect (Fig. 4c, d). Since ATG16L1 is closely related to autophagy activity, we further used western blot to examine the expression of autophagy core protein light chain 3 (LC3) in iTreg after treatment. Consistent with ATG16L1, the ratio of LC3-II/LC3-I, which indicates cell autophagy activity, in the inhibitor group was higher than that in the other groups, while the mimic group played the opposite role (Fig. 4e, f). These results suggested that miR-142-3p may improve iTreg autophagy and Foxp3 expression through the miR-1423p-ATG16L1-Foxp3 axis, as previously described for tTregs $^{13}$.

Knockdown of miR-142-3p enhances iTreg anti-apoptotic ability through promotion of demethylation of $\mathrm{H} 3 \mathrm{~K} 27 \mathrm{me} 3$ via KDM6A targeting

Trimethylation of H3K27 causes gene silencing and transcriptional repression ${ }^{5}$. KDM6B (Jmjd3) and KDM6A are histone demethylases that can specifically promote the demethylation of $\mathrm{H} 3 \mathrm{~K} 27 \mathrm{me} 3$. Bcl-2 participates in the anti-apoptotic ability and function of T lymphocytes ${ }^{33,34}$. Knockdown of miR-142-3p promoted the expression of Bcl-2 protein and Bcl-2 mRNA in iTregs (Fig. 5a-c). However, these changes were reversed by the addition of 


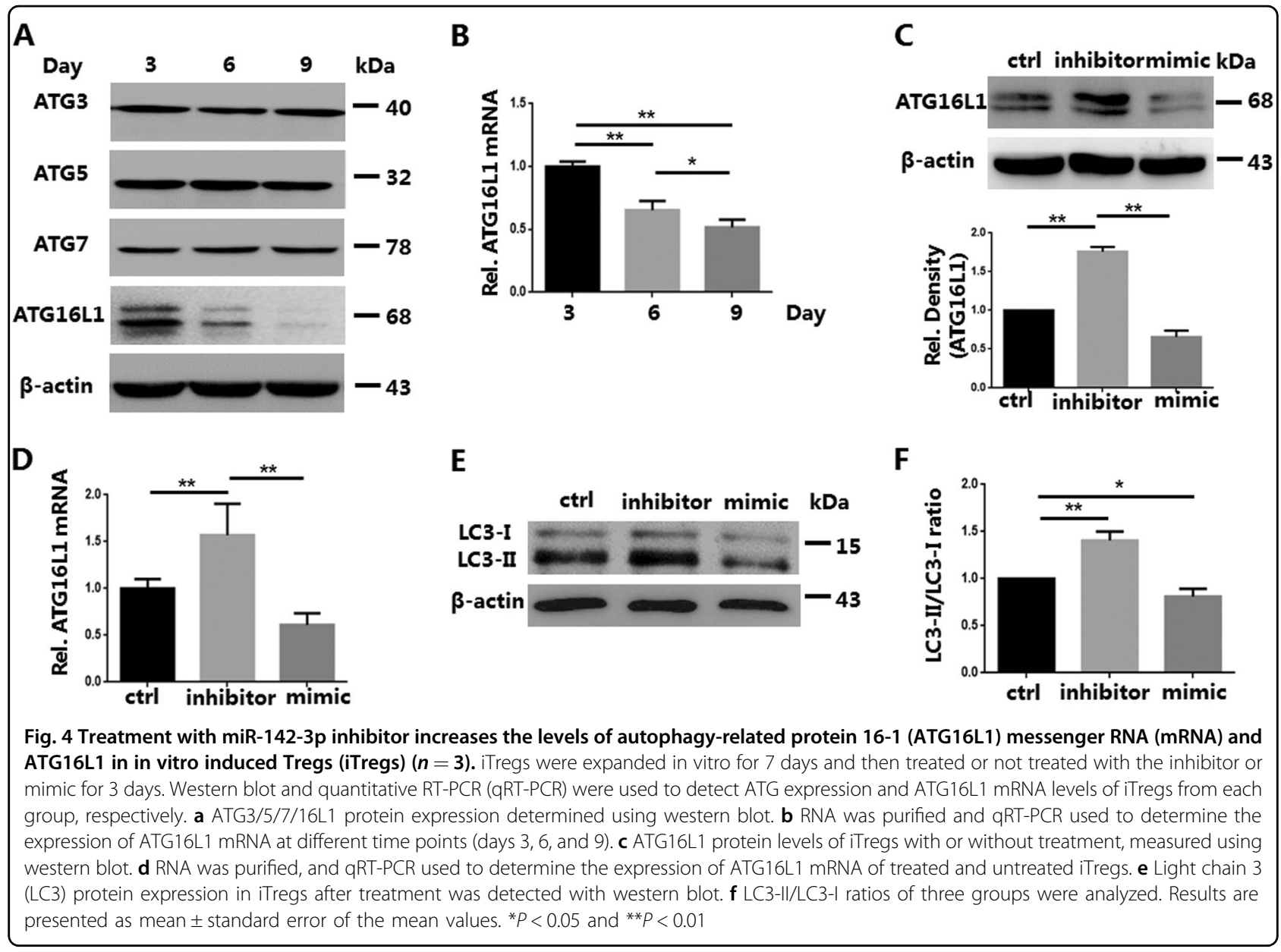

KDM6A inhibitor GSK J4 to the miR-142-3p inhibitor group (Fig. 5d). Therefore, we hypothesized that miR-142$3 \mathrm{p}$ affected H3K27 modification by targeting KDM6A, and thereby regulated $\mathrm{Bcl}-2$ expression.

TargetScan (targetscan.org), MIRDB (http://www. mirdb.org/), and microRNA (http://www.microrna.org/) are the three most commonly used and most reliable miRNA target gene prediction software applications. The three software programs predicted that 6-8 nucleosides at the $5^{\prime}$ ends of miR-142-3P could bind to the $3^{\prime}$-UTR of KDM6A. The luciferase assay of KDM6A revealed the potential binding sites of miR-142-3p. miR-142-3p significantly decreased the fluorescence intensity of KDM6A, compared with the control group and the mutant group (Fig. 5e).

Western blot assay also found that miR-142-3p affected the expression of KDM6A in iTregs (Fig. 5f). We then tested whether H3K27me3 expression in iTregs changed after treatment with a miR-142-3p inhibitor. The results indicated that H3K27me3 expression was reduced in the inhibitor group (Fig. 5g). These results revealed that miR142-3p could directly bind to KDM6A and negatively regulate KDM6A expression. Thus, knockdown of miR142-3p promoted KDM6A expression, which resulted in increased demethylation of $\mathrm{H} 3 \mathrm{~K} 27 \mathrm{me} 3$, reduced the transcriptional repression of Bcl-2, and enhanced the antiapoptotic ability and function of iTregs.

In an in vivo model, down-regulation of miR-142-3p significantly promotes mouse survival and inhibits GVHD progression

Knockdown of miR-142-3p promoted iTreg expansion, Foxp3 expression, and anti-apoptotic ability in vitro. To determine whether these changes improved iTreg immunosuppressive function in a GVHD model, human iTregs $\left(10 \times 10^{6}\right)$ after treatment were injected with PBMC $\left(10 \times 10^{6} ; 1: 1\right.$ ratio $)$ into highly immunodeficient NCG mice. All three groups that received transferred iTregs had significantly reduced GVHD-induced mortality, compared with the mice receiving PBMCs only (Fig. 6a). The inhibitor group had significantly greater prolonged survival and an apparent delay in weight loss, compared with the control and mimic groups. While the 


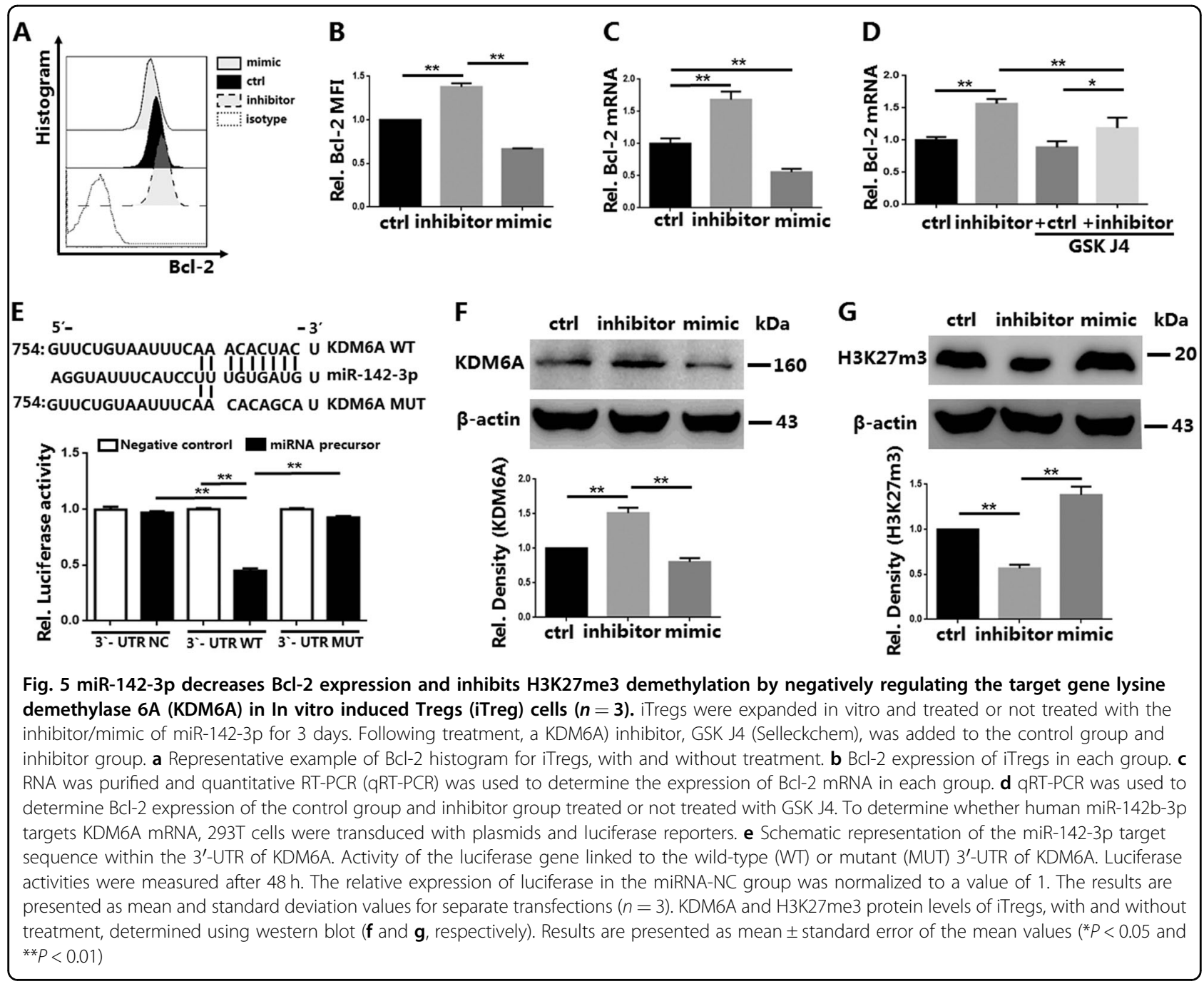

mimic group had even weaker protection against GVHD (Fig. 6b).

We also evaluated a clinical indicator of GVHD in the mice; the clinical score included weight loss, activity, fur texture, posture, and skin integrity ${ }^{35}$. The results indicated that the inhibitor group had fewer clinical symptoms of GVHD compared with the other group (Fig. 6c). During the pathophysiological process of GVHD, multiple organs (e.g., liver, kidney, and intestine) suffer tissue damage and functional failure ${ }^{36,37}$. Therefore, we performed HE staining of tissues; the mice were humanely killed on day 21 . The results for the mice treated with phosphate-buffered saline were normal; the other four GVHD groups had different degrees of pathological change (Fig. 6d). Severe tissue damage and numerous inflammatory cells were found in the livers, intestines, and kidneys from the PBMC group mice. Compared with the control group mice, the mimic group animals had apparent hepatocyte damage, glomerular destruction, and intestinal mucosal dissolution. These pathological changes were alleviated in the inhibitor group mice; there was some local inflammatory response, but no significant tissue damage.

\section{Knockdown of miR-142-3p promotes the survival and suppressive function of iTregs in vivo}

To further examine the survival and function of iTregs after transfer into the GVHD model mice, we used the same HLA mismatch strategy used in our previous study $^{38}$. We used HLA-A2- iTreg and HLA-A2+ PBMC mismatching to track the status of iTregs in vivo. Up to $100 \mu \mathrm{l}$ of PB was collected from mice in the different groups on specified dates. On day 7 after infusion, the CD45+ cells in the blood samples from the PBMC-only group were almost entirely HLA-A2+ PBMCs; no HLAA2- iTregs were detected (Fig. 7a, b). The ratio of iTregs in the inhibitor group was higher than that in the other two groups. Consistent with the in vitro results, further 


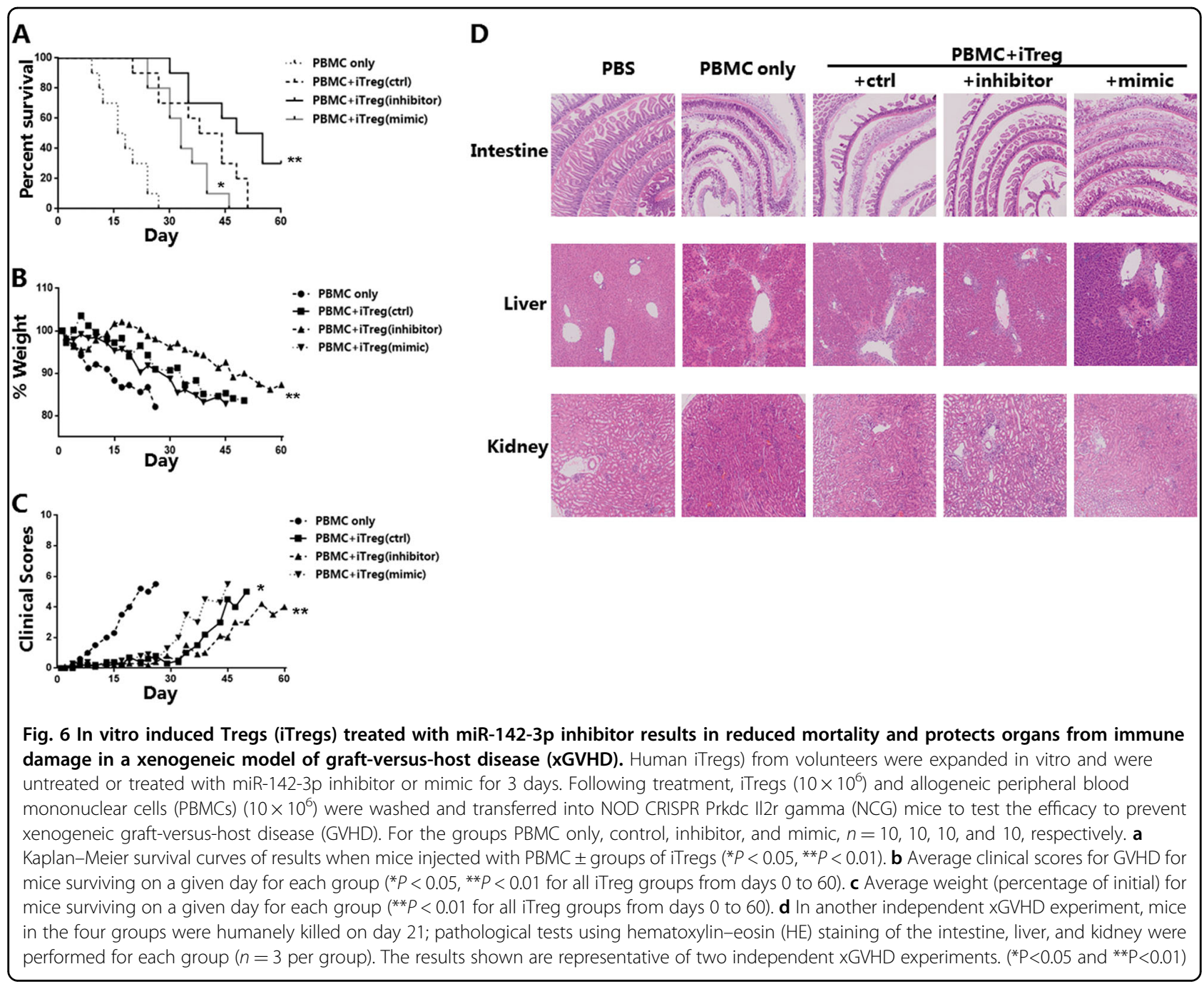

analysis of Foxp3 expression revealed that the inhibitor group had higher expression; there was no statistically significant difference in Foxp3 expression in the mimic group compared with the control group (Fig. 7c).

During GVHD development, CD8+ T cells have an important role in the immunopathological mechanism ${ }^{39}$. However, iTregs can inhibit the proliferation and function of CD8+ $\mathrm{T}$ cells, which is the basic principle of Treg adoptive cell therapy for $\mathrm{GVHD}^{40}$. The results, which are presented in Fig. 7d, indicated that CD45+HLA-A2cells were rare in the $\mathrm{PB}$ of the end-stage mice in the noninhibitor groups. There were few $(12.71 \pm 1.32 \%)$ cells in the inhibitor group, which indicated enhanced antiapoptotic ability and survival. The results also indicated that the inhibitor group had fewer CD45+HLA-A2+ cells compared with the other groups (Fig. 7e). Subtype analysis of the CD45+HLA $\gamma-\mathrm{A} 2+$ cells revealed that CD8+ $\mathrm{T}$ cells were the most significantly suppressed cell type in the inhibitor group (Fig. 7f). The mice in the inhibitor group had the most significant and prolonged suppression of CD8+ T cells.

\section{miR-142-3p has effects on the infiltration of iTregs and inflammatory cells in different organs of the xenogeneic GVHD model mice}

The HE staining results revealed that knockdown of miR-142-3p enhanced iTreg protection against immune response-associated destruction of GVHD target organs. We bound human monoclonal antibodies against HLAA2 and Foxp3 to the secondary antibodies Alexa Fluor 488 and Alexa Fluor 647, respectively. We then detected CD45+HLA-A2+ PBMCs (Alexa Fluor 488+) and CD45 +HLA-A2-Foxp3+ iTregs (Alexa Fluor 647+) in target organs on day 21 using immunofluorescence (Fig. 8a-C). Infiltration of Alexa Fluor 488+ PBMCs and Alexa Fluor $647+$ iTregs differed in different groups and different target organs. Consistent with the results of other studies, we found that Alexa Fluor 488+ PBMC infiltration was 


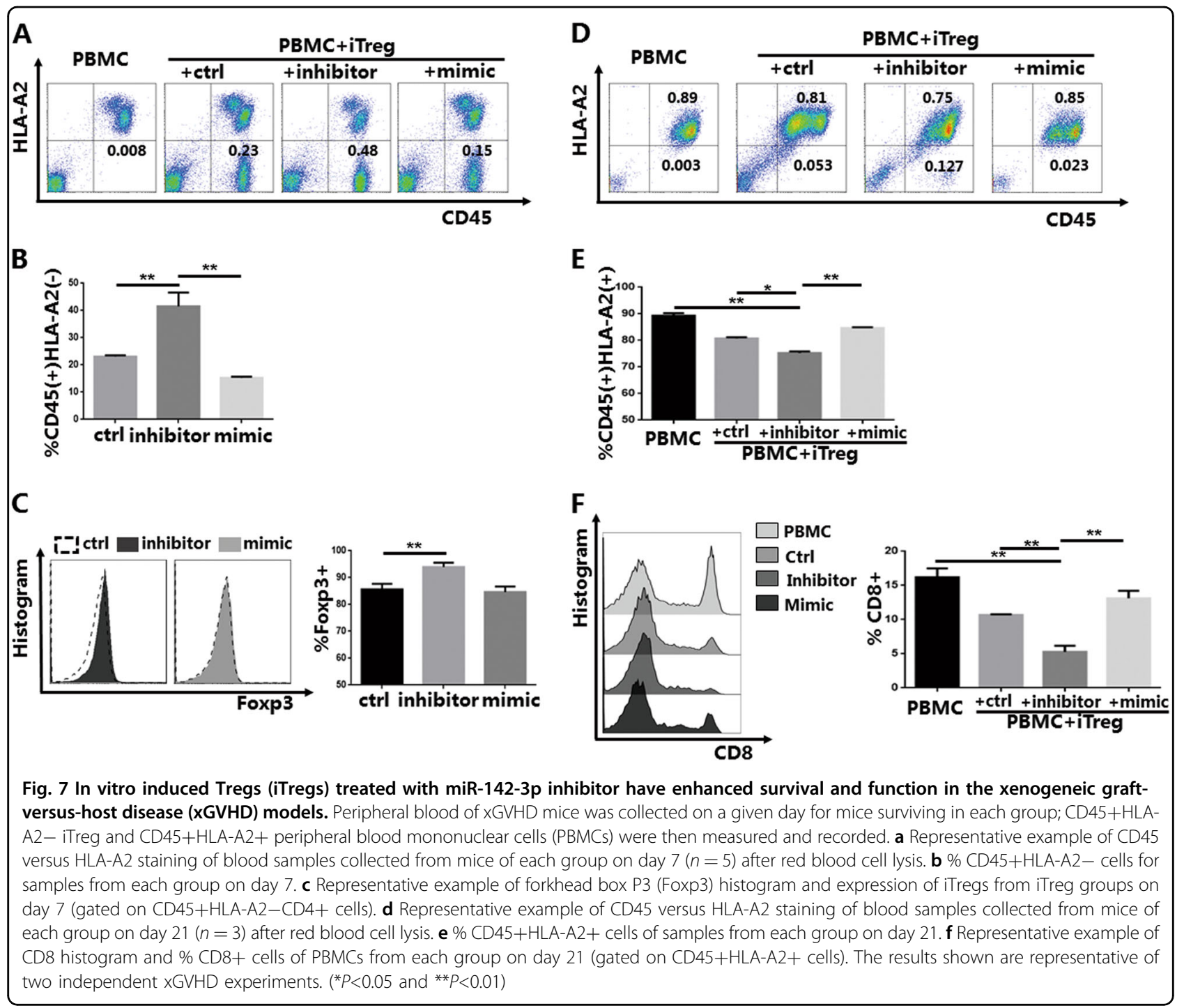

most pronounced in the intestine, compared with the liver and kidney ${ }^{37,41}$; there were no statistically significant differences in inflammatory infiltration between liver and kidney (Fig. 8d). Compared with Alexa Fluor 488+ inflammatory cells, the number of Alexa Fluor 647+ iTregs detected in the liver and kidney was over several times greater (liver: 5.5 times; kidney: 2.5 times). Compared with the other groups, knockdown of miR-142-3p resulted in a higher Alexa Fluor 647+ iTreg proportion in all three target organs (Fig. 8e). This result indicated that knockdown of miR-142-3p enhanced the anti-apoptotic ability and survival of iTregs in vivo and that it may also affect the migration of iTregs to GVHD target organs.

\section{Discussion}

In autoimmune diseases, self-antigen recognition disorders and dysfunction of immune tolerance results in immune attack of $\mathrm{CD}^{+}{ }^{+}$or $\mathrm{CD}^{+} \mathrm{T}$ cells to self-antigens.
This response damages target tissues ${ }^{42}$. The Treg is one of the immunosuppressive factors in humans, and Treg clinical trials are confronted by challenges ${ }^{43}$ : (1) Tregs in PB (especially tTregs) are too rare to easily extract sufficient numbers, and (2) the proliferative capacity, survival, and function of Tregs need further study. miR-142-3p expression is elevated in the $\mathrm{PB}$ of patients with multiple sclerosis ${ }^{44}$. At the same time, miR-142-3p regulates the expression of intracellular ATP in regulatory $\mathrm{T}$ cells ${ }^{45}$. Therefore, miR-142-3p may be a potential target for modulating the biological activity of Treg cells.

miRNA regulates the proliferation, Foxp3 expression, and function of tTregs and iTregs through multiple pathways. For example, miR-146a decreases suppressor function by targeting Stat 1 in tTregs ${ }^{46}$. miR-126 increases the induction and suppressive function of iTregs via the PI3K-Akt pathway ${ }^{47}$. Knockdown of miR-142-3p increases the expression of AC9 and cAMP in Tregs, causing 


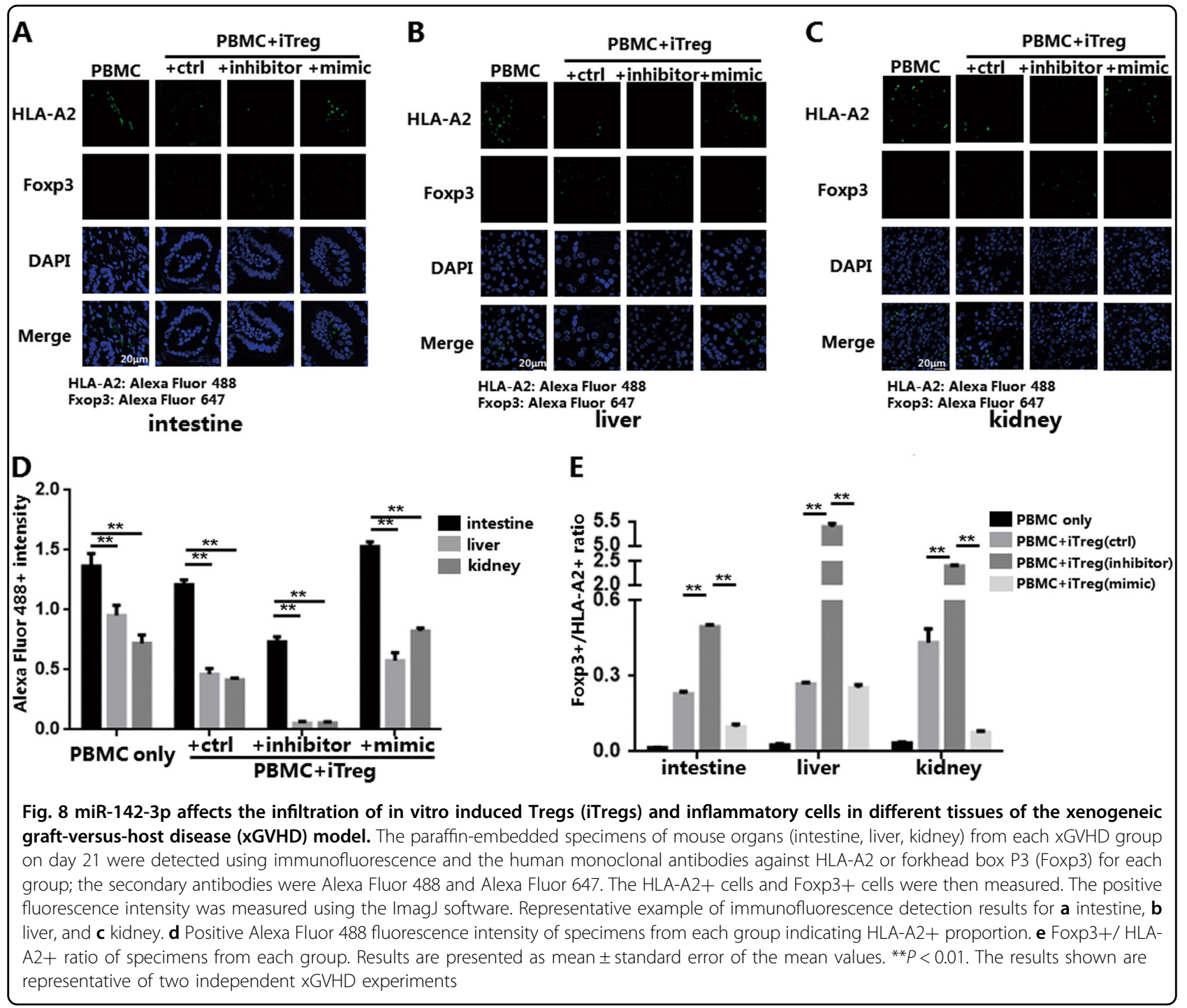

decreased intracellular ATP levels, and upregulation of the functionally related protein Foxp3 expression ${ }^{45,48}$. miR-142-3p levels are independent of Foxp3 expression, but capable of positively or negatively regulating cell proliferation and survival ${ }^{49}$. However, no studies have examined the anti-apoptotic ability of human iTregs. Successful clinical application of the use of iTregs must also solve the problems associated with cell proliferation and apoptosis in iTreg culture in vitro and the resulting limited numbers of active cells ${ }^{50,51}$. Our study is the first to find that except for proliferation and Foxp3 expression, miR-142-3p regulated the anti-apoptotic ability of iTreg by mediating histone modification.

We found that after iTregs were cultured for 7 days in vitro, the proliferative capacity began to decrease. However, knockdown of miR-142-3p reversed these changes and increased the expression of Ki67 in the iTregs. We also found that miR-142-3p knockdown improved Foxp3 expression in iTregs; Foxp3 is the most important protein associated with the suppressor function of iTregs ${ }^{52}$. In addition to the core protein, Foxp3, iTreg also inhibits inflammation and immune regulation by secreting large amounts of anti-inflammation cytokines ${ }^{17,18}$. iTreg differentiation impaired by the increased production of TNF- $\alpha$ is associated with the inflammation development in experimental autoimmune encephalomyelitis ${ }^{53}$. TNF- $\alpha$ can inhibit Smad3 phosphorylation by inducing TNFR2 expression and activating AKT, resulting in repressed iTreg differentiation, decreased expression of Foxp3, and impaired function ${ }^{54,55}$. Foxp3+ iTreg expressed lower IFN- $\gamma$ and TNF- $\alpha$, compared with Fox$\mathrm{p} 3^{\text {low/- }}$ iTreg $^{56}$. Meanwhile, blockage of C3a/C5a reduces the secretion of IFN- $\gamma$ and TNF- $\alpha$ in iTreg and enhanced the suppressive function in GVHD ${ }^{57}$. Knockdown of miR142-3p significantly decreased the expression of TNF- $\alpha$ and IFN- $\gamma$. However, miR-142-3p overexpression did not 
promote the expression of TNF- $\alpha$ or IFN- $\gamma$, it repressed the expression of TGF- $\beta$ and IL-10.

As an autophagy-related protein, ATG16L1 controls cell survival and function by regulating the autophagy state during the development of $\mathrm{T}$ lymphocytes ${ }^{58}$. ATG16L1 also regulates $\mathrm{T}$ cell activation and differentiation in some autoimmune diseases, such as inflammatory bowel disease and $\operatorname{SLE}^{59,60}$. Similar to the tTregs $^{13}$, only ATG16L1 expression changed; it decreased during iTreg culture. We also found that treatment with a miR-142-3p inhibitor reversed the changes. Consistent with the change of ATG16L1, knockdown of miR-142-3p increased LC3-II/LC3-I ratio in iTreg, indicating strengthened autophagy activity. This result suggested that similar to the tTregs, knockdown of miR-142-3p regulates the function and autophagy of iTreg via the miR-142-3p-ATG16L1-Foxp3 axis.

Histone modification is an important mechanism regulating gene expression. Histone modification is also closely related to $\mathrm{T}$ cell proliferation and apoptosis ${ }^{61}$. We found that miR-142-3p regulated the methylation status of histones in iTregs. Deletion of KDM6A results in a decrease in $\mathrm{PB} T$ cells $^{62}$. In multiple cell types, aggregation of $\mathrm{H} 3 \mathrm{~K} 27 \mathrm{me} 3$ at the promoter of $\mathrm{Bcl}-2$ induces cell apoptosis, 93,64 . We found that miR-142-3p negatively regulated the expression of the target gene KDM6A, which in turn modulated the demethylation status of H3K27me3 in iTregs. At the same time, miR-142-3p inhibited Bcl-2 expression in the iTregs. These results indicated that we can inhibit miR-142-3p to enhance the demethylation of H3K27me3 by targeting KDM6A, thereby upregulating the expression of Bcl-2 in iTregs and enhancing the anti-apoptotic ability of iTregs, both in vivo and in vitro.

However, the clinical application of iTreg use is hindered by the challenges associated with maintaining sustained high levels of Foxp3 and Treg survival in vivo ${ }^{51}$. Consistent with the in vitro results, knockdown of miR-142-3p increased Foxp3 expression and survival of iTregs in vivo. As the stable survival of iTreg, both in vivo and in vitro, is essential for adoptive therapy, we found knockdown of miR-142-3p enhanced the autophagy activity and antiapoptotic ability of iTreg, resulting in prolonged survival. This demonstrates that prolonged survival of iTreg can better exert the therapeutic effect of GVHD. Subsequently, the pathological examination that included HE staining and immunofluorescence assay revealed that knockdown of miR-142-3p enhanced the anti-apoptotic ability of iTregs, prolonged the survival in vivo, and increased protection against GVHD. miR-142-3p may also be involved in the regulation of iTreg migration.

In summary, this study is the first to find that addition of a miR-142-3p inhibitor to iTregs cultured in vitro enhanced iTreg resistance with increased anti-apoptotic ability, immunosuppressive function, and maintained Foxp3 expression both in vivo and in vitro, through the miR-142-3p-KDM6A-H3K27me3-Bcl-2 pathway. Study of the related mechanisms of miRNA and histone modification represents a new direction for iTreg clinical trials.

\section{Acknowledgements}

This work was supported in part by grants from the Major Program of the National Natural Science Fund (81530048; X.W.), the Priority Academic Program Development of Jiangsu Higher Education Institutions Fund, the National Natural Science Fund (81571564; L.L.), and the National Natural Science Fund (81571557; Y.X.) of China.

\section{Author details}

${ }^{1}$ Hepatobiliary Center, First Affiliated Hospital, Nanjing Medical University, No. 300 Guangzhou Road, Nanjing, Jiangsu Province 210029, China. ${ }^{2}$ Department of Anesthesiology, First Affiliated Hospital, Nanjing Medical University, No. 300 Guangzhou Road, Nanjing, Jiangsu Province 210029, China

\section{Authors' contributions}

Ji Gao, Jian Gu. and X.P designed the experiments, performed the experiments, analyzed the data, and wrote the manuscript. X.G., Z.J., S.Z., and Y.X. performed the experiments and interpreted the data. L.L. designed the overall concept, analyzed the data, and wrote the manuscript. X.W.designed the overall concept, analyzed the data, and wrote the manuscript.

\section{Conflict of interest}

The authors declare that they have no conflict of interest.

\section{Publisher's note}

Springer Nature remains neutral with regard to jurisdictional claims in published maps and institutional affiliations.

Received: 19 February 2019 Revised: 25 March 2019 Accepted: 26 March 2019

Published online: 15 April 2019

\section{References}

1. Gu, J. et al. TGF-ß-induced CD4+Foxp3+ T cells attenuate acute graft-versushost disease by suppressing expansion and killing of effector CD8+ cells. J. Immunol. 193, 10 (2014).

2. Agle, $\mathrm{K}$. et al. Bim regulates the survival and suppressive capability of $\mathrm{CD} 8(+)$ FOXP3(+) regulatory T cells during murine GVHD. Blood 132, $435-447$ (2018).

3. Sharabi, A. et al. Regulatory $T$ cells in the treatment of disease. Nat. Rev. Drug Discov. (2018). [Epub ahead of print] https://doi.org/10.1038/nrd.2018.148.

4. Zhong, $\mathrm{H}$. et al. TGF-beta-Induced CD8(+)CD103(+) regulatory T cells show potent therapeutic effect on chronic graft-versus-host disease lupus by suppressing B cells. Front. Immunol. 9, 35 (2018).

5. Niederberger, E., Resch, E., Parnham, M. J. \& Geisslinger, G. Drugging the pain epigenome. Nat. Rev. Neurol. 13, 434-447 (2017).

6. Leentjens, J. et al. Trained innate immunity as a novel mechanism linking infection and the development of atherosclerosis. Circ. Res. 122, 664-669 (2018).

7. Zhan, Y., Carrington, E. M., Zhang, Y., Heinzel, S. \& Lew, A. M. Life and death of activated T cells: how are they different from naive T cells? Front. Immunol. 8, 1809 (2017).

8. Wang, X. et al. Preferential control of induced regulatory $T$ cell homeostasis via a Bim/Bcl-2 axis. Cell Death Dis. 3, e270 (2012).

9. Yang, D., Okamura, H., Teramachi, J. \& Haneji, T. Histone demethylase Jmjd3 regulates osteoblast apoptosis through targeting anti-apoptotic protein $\mathrm{BCl}-2$ and pro-apoptotic protein Bim. Biochim. Biophys. Acta 1863, 650-659 (2016).

10. Manna, S. et al. Histone H3 Lysine 27 demethylases Jmjd3 and Utx are required for T-cell differentiation. Nat. Commun. 6, 8152 (2015).

11. Hippen, K. L., Loschi, M., Nicholls, J., MacDonald, K. P. A. \& Blazar, B. R. Effects of microRNA on regulatory $T$ cells and implications for adoptive cellular therapy to ameliorate graft-versus-host disease. Front. Immunol. 9, 57 (2018). 
12. Ding, S. et al. Decreased microRNA-142-3p/5p expression causes CD4+ T cell activation and B cell hyperstimulation in systemic lupus erythematosus. Arthritis Rheum. 64, 2953-2963 (2012)

13. Lu, Y. et al. miR-142-3p regulates autophagy by targeting ATG16L1 in thymicderived regulatory T cell (tTreg). Cell Death Dis. 9, 290 (2018).

14. Hippen, K. L. et al. Umbilical cord blood regulatory T-cell expansion and functional effects of tumor necrosis factor receptor family members OX40 and 4-1BB expressed on artificial antigen-presenting cells. Blood 112, 2847-2857 (2008)

15. Schaefer, J. S. et al. MicroRNA signatures differentiate Crohn's disease from ulcerative colitis. BMC Immunol. 16, 5 (2015).

16. Godefroy, E. et al. Expression of CCR6 and CXCR6 by gut-derived CD4 $(+) / C D 8 a l p h a(+)$ T-regulatory cells, which are decreased in blood samples from patients with inflammatory bowel diseases. Gastroenterology $\mathbf{1 5 5}$ 1205-1217 (2018).

17. DeLong, C. M., Bragg, R. \& Simmons, J. A. Evidence for spatial representation of object shape by echolocating bats (Eptesicus fuscus). J. Acoust. Soc. Am. 123 4582-4598 (2008).

18. Osnes, L. T., Nakken, B., Bodolay, E. \& Szodoray, P. Assessment of intracellular cytokines and regulatory cells in patients with autoimmune diseases and primary immunodeficiencies-novel tool for diagnostics and patient followup. Autoimmun. Rev. 12, 967-971 (2013).

19. Meylan, F. \& Siegel, R. M. TNF superfamily cytokines in the promotion of Th9 differentiation and immunopathology. Semin. Immunopathol. 39, 21-28 (2017).

20. Puniya, B. L. et al. A mechanistic computational model reveals that plasticity of CD4(+) T cell differentiation is a function of cytokine composition and dosage. Front. Physiol. 9, 878 (2018).

21. Bhaumik, S. \& Basu, R. Cellular and molecular dynamics of Th17 differentiation and its developmental plasticity in the intestinal immune response. Front. Immunol. 8, 254 (2017).

22. Schmitt, $H$. et al. Expansion of $\mathrm{IL}-23$ receptor bearing TNFR2 $+\mathrm{T}$ cells is associated with molecular resistance to anti-TNF therapy in Crohn's disease Gut. 68, 814-828 (2018).

23. Croft, M. \& Siegel, R. M. Beyond TNF: TNF superfamily cytokines as targets for the treatment of rheumatic diseases. Nat. Rev. Rheumatol. 13, 217-233 (2017).

24. Mancusi, A., Piccinelli, S., Velardi, A. \& Pierini, A. The effect of TNF-alpha on regulatory $T$ cell function in graft-versus-host disease. Front. Immunol. 9, 356 (2018).

25. Levine, J. E. Implications of TNF-alpha in the pathogenesis and management of GVHD. Int. J. Hematol. 93, 571-577 (2011)

26. Mehta, N. N. et al. IFN-gamma and TNF-alpha synergism may provide a link between psoriasis and inflammatory atherogenesis. Sci. Rep. 7, 13831 (2017).

27. Yang, G., Driver, J. P. \& Van Kaer, L. The role of autophagy in iNKT cell development. Front. Immunol. 9, 2653 (2018).

28. Becher, J. et al. AMBRA1 controls regulatory T-cell differentiation and homeostasis upstream of the FOXO3-FOXP3 axis. Dev. Cell 47, 592-607 e596 (2018).

29. Rivera Vargas, T. et al. Selective degradation of PU.1 during autophagy represses the differentiation and antitumour activity of TH9 cells. Nat. Commun. 8, 559 (2017).

30. Keller, C. W. et al. The autophagy machinery restrains iNKT cell activation through CD1D1 internalization. Autophagy 13, 1025-1036 (2017).

31. Kabat, A. M. et al. The autophagy gene Atg16l1 differentially regulates Treg and $\mathrm{TH} 2$ cells to control intestinal inflammation. Elife 5, e12444 (2016).

32. Pua, H. H., Dzhagalov, I., Chuck, M., Mizushima, N. \& He, Y. W. A critical role for the autophagy gene Atg5 in T cell survival and proliferation. J. Exp. Med. 204, 25-31 (2007).

33. Tuzlak, S. et al. The BCL-2 pro-survival protein A1 is dispensable for $\mathrm{T}$ cell homeostasis on viral infection. Cell Death Differ. 24, 523-533 (2017).

34. Zhang, N., Hartig, H., Dzhagalov, I., Draper, D. \& He, Y. W. The role of apoptosis in the development and function of T lymphocytes. Cell Res. 15, 749-769 (2005)

35. al, K. R. Ce An experimental model of idiopathic pneumonia syndrome after bone marrow transplantation: I. The roles of minor $\mathrm{H}$ antigens and endotoxin. Blood 8, 3230-3239 (1996).

36. Riesner, $\mathrm{K}$. et al. Initiation of acute graft-versus-host disease by angiogenesis. Blood 129, 2021-2032 (2017).

37. Panoskaltsis-Mortari, A. et al. In vivo imaging of graft-versus-host-disease in mice. Blood 103, 3590-3598 (2004).
38. Lu, Y. et al. miR-146b antagomir-treated human Tregs acquire increased GVHD inhibitory potency. Blood 128, 1424-1435 (2016).

39. Pierini, A. et al. TNF-alpha priming enhances CD4+FoxP3+ regulatory T-cell suppressive function in murine GVHD prevention and treatment. Blood $\mathbf{1 2 8}$ 866-871 (2016).

40. Koenecke, C. et al. Alloantigen-specific de novo-induced Foxp3+ Treg revert in vivo and do not protect from experimental GVHD. Eur. J. Immunol. 39, 3091-3096 (2009).

41. Fischer, J. C. et al. RIG-I/MAVS and STING signaling promote gut integrity during irradiation- and immune-mediated tissue injury. Sci. Transl. Med. eaag2513 (2017).

42. Handel, A. E., Irani, S. R. \& Hollander, G. A. The role of thymic tolerance in CNS autoimmune disease. Nat. Rev. Neurol. 14, 723-734 (2018).

43. Tang, Q. \& Vincenti, F. Transplant trials with Tregs: perils and promises. J. Clin. Invest. 127, 2505-2512 (2017).

44. Arruda, L. C. et al. Autologous hematopoietic SCT normalizes miR-16, -155 and $-142-3 p$ expression in multiple sclerosis patients. Bone Marrow Transplant. 50 380-389 (2015)

45. Zhao, J. et al. Selective depletion of CD4+CD25+Foxp3+ regulatory T cells by low-dose cyclophosphamide is explained by reduced intracellular ATP levels. Cancer Res. 70, 4850-4858 (2010).

46. Lu, L. F. et al. Function of miR-146a in controlling Treg cell-mediated regulation of Th1 responses. Cell 142, 914-929 (2010).

47. Qin, A. et al. MicroRNA-126 regulates the induction and function of CD4(+) Foxp3(+) regulatory T cells through PI3KJAKT pathway. J. Cell. Mol. Med. 17, 252-264 (2013)

48. Huang, B. et al. miR-142-3p restricts CAMP production in CD4+CD25T cells and CD4+CD25+ TREG cells by targeting AC9 mRNA. EMBO Rep. 10, 180-185 (2009)

49. Holmstrom, K., Pedersen, A. E. \& Gad, M. Analysis of miR-146a and miR-142-3p as potential markers of freshly isolated or in vitro-expanded human Treg cells. Scand. J. Immunol. 85, 113-121 (2017).

50. Bilate, A. M. \& Lafaille, J. J. Induced CD4+Foxp3+ regulatory T cells in immune tolerance. Annu. Rev. Immunol. 30, 733-758 (2012).

51. Yang, J. et al. Adoptive cell therapy of induced regulatory $T$ cells expanded by tolerogenic dendritic cells on murine autoimmune arthritis. J. Immunol. Res. 2017, 7573154 (2017).

52. Chen, W. et al. Conversion of peripheral CD4+CD25- naive T cells to CD4 +CD25+ regulatory $T$ cells by TGF-beta induction of transcription factor Foxp3. J. Exp. Med. 198, 1875-1886 (2003).

53. Zhang, Q. et al. TNF-alpha impairs differentiation and function of TGF-betainduced Treg cells in autoimmune diseases through Akt and Smad3 signaling pathway. J. Mol. Cell. Biol. 5, 85-98 (2013).

54. Wajant, H., Pfizenmaier, K. \& Scheurich, P. Tumor necrosis factor signaling. Cell Death Differ. 10, 45-65 (2003).

55. Liu, G. et al. The receptor S1P1 overrides regulatory $T$ cell-mediated immune suppression through Akt-mTOR. Nat. Immunol. 10, 769-777 (2009).

56. Reynolds, B. C. et al. Exposure to inflammatory cytokines selectively limits GM-CSF production by induced T regulatory cells. Eur. J. Immunol. 44 3342-3352 (2014).

57. van der Touw, W. et al. Cutting edge: eceptors for C3a and C5a modulate stability of alloantigen-reactive induced regulatory T cells. J. Immunol. 190 5921-5925 (2013)

58. Le Texier, L. et al. Autophagy-dependent regulatory T cells are critical for the control of graft-versus-host disease. JCl Insight 1, e86850 (2016).

59. Liu, J. Z. et al. Association analyses identify 38 susceptibility loci for inflammatory bowel disease and highlight shared genetic risk across populations. Nat. Genet. 47, 979-986 (2015).

60. Rai, S. et al. The ATG5-binding and coiled coil domains of ATG16L1 maintain autophagy and tissue homeostasis in mice independently of the WD domain required for LC3-associated phagocytosis. Autophagy $\mathbf{1 5}$ 599-612 (2019).

61. Rothenberg, E. V. The chromatin landscape and transcription factors in T cell programming. Trends Immunol. 35, 195-204 (2014).

62. Bosselut, R. Pleiotropic functions of H3K27Me3 demethylases in immune cel differentiation. Trends Immunol. 37, 102-113 (2016).

63. Svotelis, A. et al. H3K27 demethylation by JMJD3 at a poised enhancer of antiapoptotic gene BCL2 determines ERalpha ligand dependency. EMBO J. 30, 3947-3961 (2011)

64. al, Le Targeted inhibition of histone H3K27 demethylation is effective in highrisk neuroblastoma. Sci. Transl. Med. 10, eaao4680 (2018). 